\title{
EDITORIAL
}

\section{Frailty: we need valid and reliable tools in critical care}

\author{
Hans Flaatten ${ }^{1,2^{*}}$ (1) and Andrew Clegg ${ }^{3}$
}

(c) 2018 Springer-Verlag GmbH Germany, part of Springer Nature and ESICM

The concept of frailty has been long established in geriatric medicine, but, more recently, has been gaining considerable traction in specialist settings including critical care. The development of conceptual frameworks for frailty, leading to publications on the phenotype [1] model and the cumulative deficit model [2], has provided a theoretical framework for frailty research, catalysing novel scientific investigation. The phenotype model identifies frailty on the basis of at least three of five physical characteristics (slow walking speed, impaired grip strength, low activity, unintended weight loss, and exhaustion), and the cumulative deficit model identifies frailty on the basis of a range of health deficits (signs, symptoms, diseases, disabilities, impairments), enabling calculation of a frailty index (FI) score.

There is now broad international agreement that frailty is a condition characterised by loss of biological reserves, failure of homeostatic mechanisms and vulnerability to a range of adverse outcomes, such as falls, disability, hospitalisation, cognitive decline and requirement for care [3]. It is recognised that people living with frailty typically have a range of physical, cognitive and psychosocial problems that contribute to development of the condition and adverse outcomes.

Further work to develop and validate simple tools for the assessment of frailty brought the concept closer to clinical practice [4]. More recent UK research has applied the cumulative deficit model to develop and validate an electronic frailty index (eFI) using routinely available primary care electronic health record data to identify frailty, reducing the need for a resource intensive clinical

*Correspondence: hans.flaatten@uib.no

${ }^{1}$ Department of Anaesthesia and Intensive Care, Haukeland University Hospital, Bergen, Norway

Full author information is available at the end of the article assessment [5]. A similar approach has now also been taken using hospital records to develop a hospital frailty risk score (HFRS) [6], which opens novel avenues for research, including in critical care.

Assessment of frailty presents problems in the context of acute care because most patients have never previously been evaluated for the presence of the condition in a formal way and may not be capable of a detailed assessment at admission. Hence, different and more simple methods to identify frailty must be used. Most often, the clinical frailty scale (CFS) [7] has been used. The CFS is a nine-item frailty measure that uses pictographs and clinical descriptions ranging from fit to very severe frailty and terminally ill. It enables practitioners to rapidly screen for and severity-grade frailty, even in the context of critical illness. All eight studies included in a 2018 systematic review reporting frailty assessment in ICU patients used a version of the CFS [8]. Three studies also used other methods, i.e. frailty phenotype assessment or a comprehensive geriatric assessment.

However, a key question arises regarding whether it is possible to compare results between studies given the large differences in methods of frailty identification. An important additional issue is that the feasibility of frailty identification in the critical care setting often relies on information from family or next-of-kin. An attractive feature of the CFS is the combination of simple text and a figure to stage frailty. It looks intuitive, but does this simple index really reflect frailty measured using more comprehensive methods?

Recent studies show a large variation of frailty in critically ill patients, even when the same instrument is used (Fig. 1). The CFS was used in eight studies, and frailty was reported from 13 to 53\% with no clear relationship between mean age and proportion of frailty. There may be several reasons for this finding. Many studies are small and often from a single ICU, with substantial differences

\section{6 Springer}




\section{Frailty vs mean age in ICU cohorts}

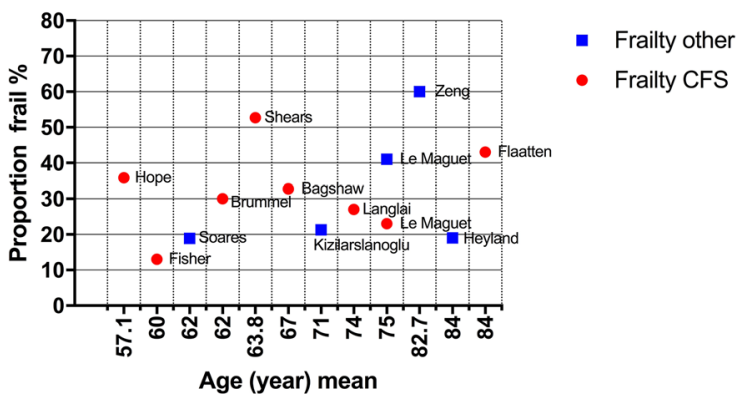

Fig. 1 The relationship between mean age in 12 ICU cohorts against the proportion of frailty in recent publications

in cohort age inclusion criteria. The geographical spread is large and the range of findings could potentially reflect the different critical care service delivery models in operation internationally.

Considered collectively, these data indicate insufficient knowledge about how to best measure and describe frailty in the critical care setting where more refined methods like a comprehensive geriatric assessment [9] from a geriatrician is highly resource-intensive and frequently unavailable.

Future studies must focus on the validity, reliability and feasibility of frailty measures. Construct validity is the degree to which a test measures the concept it is claiming to measure. Convergent validity is a component of construct validity and is a measure of the degree to which two measures of the same construct are related. This is highly important in the context of frailty in critical care, but there has been a relative absence of research in this area. Criterion validity is the extent to which a measure is related to an outcome, and can be subdivided into concurrent validity (how the measure predicts an outcome measured at the same time, such as disability) and predictive validity (how the measure predicts a future event, such as mortality). There is a strong link between frailty, co-morbidity and disability. This has historically been considered a challenge, but measurement of concurrent validity (e.g. how frailty predicts existing disability) and predictive validity (e.g. how frailty predicts future disability, nursing home admission, or mortality) are attractive options for research, particularly as studies of predictive validity have tended to focus on mortality as an outcome [10]. In addition, the inter-rater and test-retest reliability of frailty measures must be measured and reported. Such studies have started to appear [11]. Of the available tools, the CFS has been tested against other frailty instruments [12] and has now been translated to several languages and has been used extensively, not only within intensive care. It is easy to learn, but several validity and reliability properties of CSF are at present missing.

In this issue of $I C M$, a large register-based study from Brazil is published using a modified frailty index (mFI) [13]. The mFI is derived from the original FI that was developed as a part of the Canadian Study of Health and Ageing [14], and is constructed of 70 items most of them based on symptoms that describes frailty. Further modification with a reduction of the items in the FI has been used in clinical and administrative databases. This $\mathrm{mFI}$ ends up with a very small number of items and both 11- and 19-item versions have been used $[15,16]$. The 11-item mFI results in a large reduction in specific frailty deficits, leaving only 1 item, on physical function. In addition, the reduction has been carried out without a formal investigation of convergent validity against the original FI. Overall, the mFI deviates from the general multidimensional concept of frailty developed over recent decades, and is more consistent with a comorbidity measure.

Given the importance of frailty in the context of critical care, we must be confident that the instruments used to identify the condition are robust and properly validated. A wide range of tools to identify frailty are available in general settings, and should be considered for investigation in the context of critical care. In particular, the documentation of construct and criterion validity alongside reliability and feasibility for practice is necessary before we can recommended them for use in routine clinical practice.

\section{Author details \\ ${ }^{1}$ Department of Anaesthesia and Intensive Care, Haukeland University Hospi- tal, Bergen, Norway. ${ }^{2}$ Department of Clinical Medicine, University of Bergen, Bergen, Norway. ${ }^{3}$ Academic Unit of Elderly Care and Rehabilitation, University of Leeds, Bradford Teaching Hospitals NHS Foundation Trust, Bradford, UK.}

Received: 6 September 2018 Accepted: 1 October 2018

Published online: 11 October 2018

\section{References}

1. Fried LP, Tangen CM, Watson J et al (2001) Frailty in older adults: evidence for a phenotype. J Gerontol A 56:M146-M156

2. Mitnitski AB, Mogilner AJ, Rockwood K (2001) Accumulation of deficits as a proxy measure of aging. Sci World J 1:323-336. https://doi.org/10.1100/ tsw.2001.58

3. Abellan van Kan $\mathrm{G}$, Rolland Y, Bergman $\mathrm{H}$ et al (2008) The I.A.N.A task force on frailty assessment of older people in clinical practice. J Nutr Health Aging 12:29-37

4. Clegg A, Rogers LS, Young J (2015) Diagnostic test accuracy of simple instruments for identifying frailty in community-dwelling older people: a systematic review. Age Ageing 44:148-152. https://doi.org/10.1093/agein g/afu157

5. Clegg A, Bates C, Young J, Ryan R et al (2016) Development and validation of an electronic frailty index using routine primary care electronic health record data. Age Ageing 45:353-360. https://doi.org/10.1093/ ageing/afw039 
6. Gilbert T, Neuburger J, Kraindler J et al (2018) Development and validation of a Hospital Frailty Risk Score focusing on older people in acute care settings using electronic hospital records: an observational study. Lancet 391:1775-1782. https://doi.org/10.1016/S0140-6736(18)30668-8

7. Rockwood K, Song X, MacKnight C et al (2005) A global clinical measure of fitness and frailty in elderly people. CMAJ 173:489-495. https://doi. org/10.1503/cmaj.050051

8. Pugh RJ, Ellison A, Pye K et al (2018) Feasibility and reliability of frailty assessment in the critically ill: a systematic review. Crit Care 22:49. https:// doi.org/10.1186/s13054-018-1953-9

9. Welsh TJ, Gordon AL, Gladman JR (2014) Comprehensive geriatric assessment-a guide for the non-specialist. Int J Clin Pract 68:290-293. https://doi.org/10.1111/ijcp.12313

10. Muscedere J, Waters B, Varambally A et al (2017) The impact of frailty on intensive care unit outcomes: a systematic review and meta-analysis. Int Care Med 43:1 105-1122. https://doi.org/10.1007/s00134-017-4867-0

11. Shears M, Takaoka A, Rochwerg B et al (2018) Assessing frailty in the intensive care unit: a reliability and validity study. J Crit Care 45:197-203. https://doi.org/10.1016/j.jcrc.2018.02.004
12. Hope A, Hsieh SJ, Petti A et al (2017) Assessing the usefulness and validity of frailty markers in critically ill adults. Ann Am Thorac Soc 14:952-959. https://doi.org/10.1513/AnnalsATS.201607-5380C

13. Zampieri FG, Iwashyna TJ, Viglianti EM et al (2018) Association of frailty with short-term outcomes, organ support and resource use in critically ill patients. Intensive Care Med 44:1512-1520. https://doi.org/10.1007/ s00134-018-5342-2

14. Rockwood K, Song X, Macknight C et al (2005) A global clinical measure of fitness and frailty in elderly people. CMAJ 173:489-495. https://doi. org/10.1503/cmaj.050051

15. Patel KV, Brennan KL, Brennan ML et al (2014) Association of a modified frailty index with mortality after femoral neck fracture in patients aged 60 years and older. Clin Orthop Relat Res 472:1010-1017. https://doi. org/10.1007/s11999-013-3334-7

16. Velanovich V, Antoine H, Swartz A (2013) Accumulating deficits model of frailty and postoperative mortality and morbidity: its application to a national database. J Surg Res 183:104-110. https://doi.org/10.1016/j. jss.2013.01.021 